\title{
Fresnel and Fourier digital holography architectures: a comparison.
}

\author{
Damien P. , David S. Monaghan, Nitesh Pandey, \\ Bryan M. Hennelly. \\ Department of Computer Science, \\ National University of Ireland, Maynooth, \\ Co. Kildare, Ireland.
}

\section{Introduction}

In this manuscript we examine the characteristics of holograms that are captured using both Fresnel and lens-less Fourier digital holographic systems. We begin by introducing some of the fundamental equations describing the intensity distribution captured by the camera. Naturally this captured intensity will vary depending on whether the system used is a Fourier or a Fresnel due to the different reference field in each case, however as we shall see with appropriate numerical processing it is possible to obtain similar performance from both systems. We discuss a reconstruction algorithm for changing the focus depth in Fourier holograms and examine how it effects the twin image and dc terms. A theoretical comparison with Fresnel holograms is made. Experimental results are provided to support our analysis. We finish with a brief conclusion.

\section{Theory and Experimental Results}

A typical optical setup for capturing both Fresnel and lens-less Fourier digital holograms was built. The object is illuminated with a plane wave. Light scattered from this object then propagates to the camera plane where it is combined with our reference wave and the resulting intensity pattern recorded. A lens in the reference arm is used to vary the curvature of the reference field and may be removed in order to capture a Fresnel hologram. The intensity recorded by the camera may be expressed as [1]

$$
H(x)=\left|u_{\mathrm{z}}(x)+u_{\mathrm{R}}(x)\right|^{2}
$$




$$
H(x)=I_{\mathrm{z}}+I_{\mathrm{R}}+u_{\mathrm{z}}(x) u_{R}^{*}(x)+u_{\mathrm{z}}^{*}(x) u_{\mathrm{R}}(x)
$$

respectively, while the latter two terms in Eq. $(1 \mathrm{~b})$ correspond to the real and twin image fields. We use a lens of focal length, $f$, to produce a spherical reference field $u_{\mathrm{R}}(x)$ for our Fourier holography setup,

$$
u_{\mathrm{R}}(x)=\exp \left(\frac{j \pi x^{2}}{\lambda f}\right)
$$

wheras for Fresnel holography the reference is a flat unit amplitude plane wave and can be written as

$$
u_{\mathrm{R}}(x)=\exp (j \alpha)
$$

where $\alpha$ is some arbitrary constant phase. We may relate the field $u_{\mathrm{z}}(x)$ to the field in the object plane, $U(X)$ using a Fresnel transform which we define as,

$$
\begin{gathered}
u_{\mathrm{z}}(x)=\mathfrak{I}_{\mathrm{z}}\{U(X)\}(x) \\
u_{\mathrm{z}}(x)=\exp \left(\frac{j \pi x^{2}}{\lambda z}\right) \int U(X) \exp \left(\frac{j \pi x^{2}}{\lambda z}\right) \exp \left(\frac{-j 2 \pi x X}{\lambda z}\right) d X
\end{gathered}
$$

Note that for simplicity in this manuscript we ignore: (i) the finite extent of the camera, (ii) the reduction in power of higher spatial frequencies due to an averaging introduced by the finite pixel size, and (iii) sampling introduced by pixels spaced uniformly at fixed intervals from each other $[1,2]$.

We now wish to consider numerical reconstruction of both Fourier and Fresnel holograms. We may recover our Fresnel hologram numerically using the Direct Method [3]. This entails multiplying $H(x)$ by a quadratic phase factor, $\exp \left(j \pi x^{2} / \lambda z_{\mathrm{R}}\right)$, (where $z_{\mathrm{R}}$ is the desired reconstruction distance), and performing a numerical Fourier transform on the result. By varying $z_{\mathrm{R}}$ we can change the reconstruction distance. Performing such a numerical operation on the real image term from Eq. $(1 \mathrm{~b})$ produces the following result, see Fig. 1. The image in Fig. 1 corresponds to a Fresnel hologram 


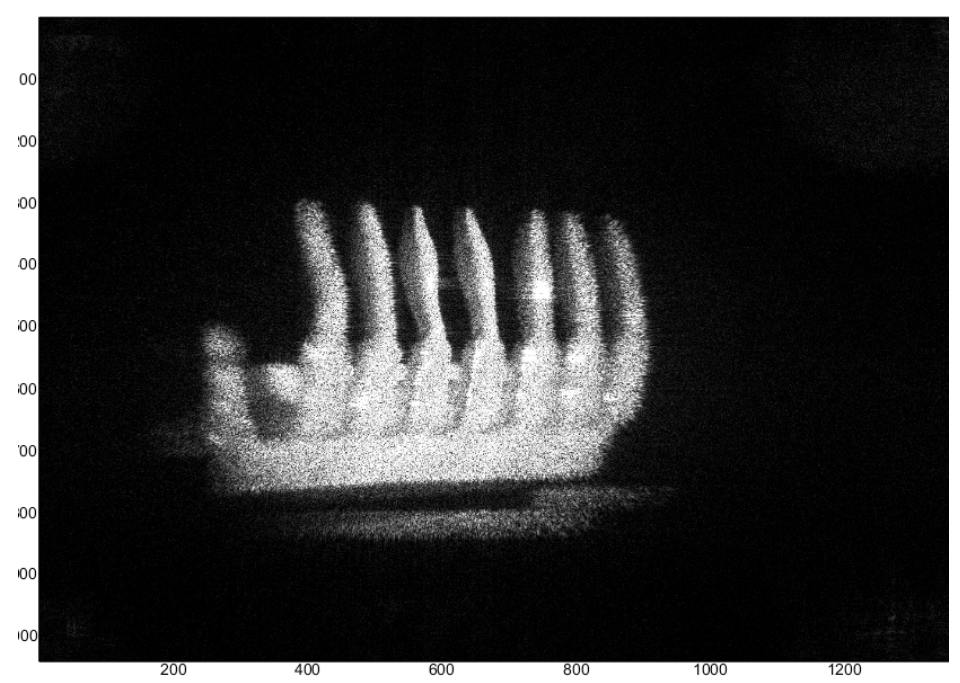

Fig. 1. Numerical reconstruction of a digital hologram captured using a Fresnel geometry. Both the DC terms and the twin image have been removed in this example using a PSI technique.

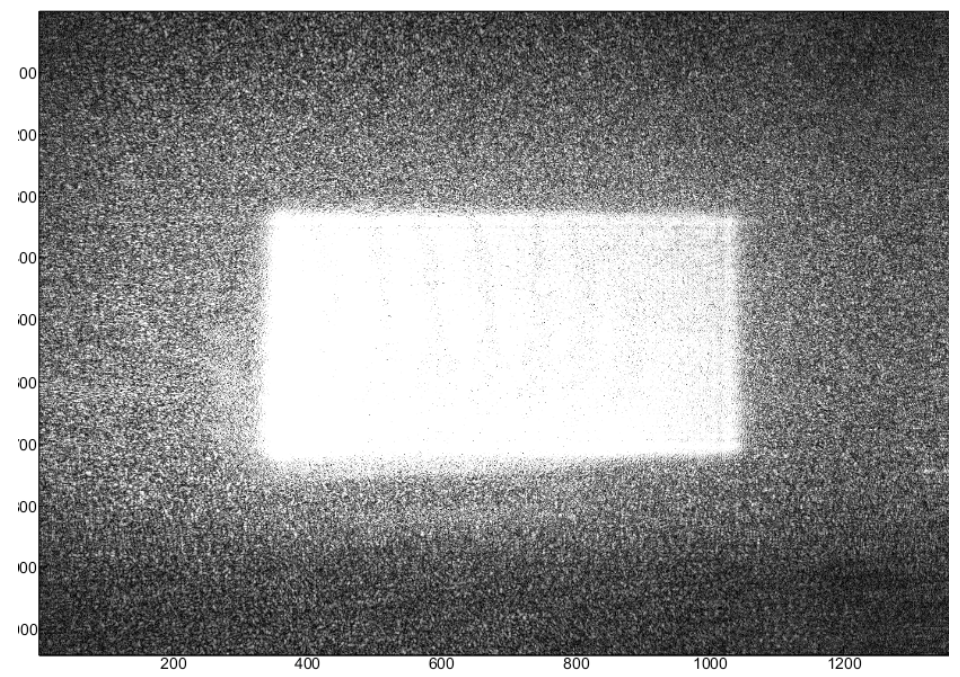

Fig. 2. Numerical reconstruction of a digital hologram captured using a Fresnel geometry. In this case the neither the twin nor DC terms have been removed and both act to significantly reduce the quality of the reconstructed hologram.

where the twin image and DC terms have been removed using a phase shifting interferometric technique (PSI). If the twin image term is not removed from Eq. $(1 \mathrm{~b})$ we see that it overlaps with the real image in the 
reconstruction plane reducing the quality of the reconstructed hologram. This can be seen in Fig. 2. Note the DC terms will also contribute negatively to the image quality. We now wish to examine how we may reconstruct our real image term [term 3 in Eq. $(1 \mathrm{~b})$ ] assuming that our incident reference field is now described by Eq. (2 a),

$$
\begin{aligned}
u_{\mathrm{z}} u_{\mathrm{R}}^{*}=\exp \left[\frac{j \pi x^{2}}{\lambda}(\right. & \left.\left.\frac{1}{z}-\frac{1}{f}-\frac{1}{z_{\mathrm{R}}}\right)\right] \\
& \times \int U(X) \exp \left(\frac{j \pi x^{2}}{\lambda z}\right) \exp \left(\frac{-j 2 \pi x X}{\lambda z}\right) d X
\end{aligned}
$$

If $z=f$ in the equation above (we set $z_{\mathrm{R}}=0$ for the moment) then the leading quadratic phase term drops out and we can relate $u_{\mathrm{z}} u_{\mathrm{R}}^{*}$ to $U(X) \exp \left(j \pi x^{2} / \lambda z\right)$ by a Fourier transform. A similar analysis holds for the twin term. In Fig. 3 we present a typical Fourier reconstruction, see figure caption for details.

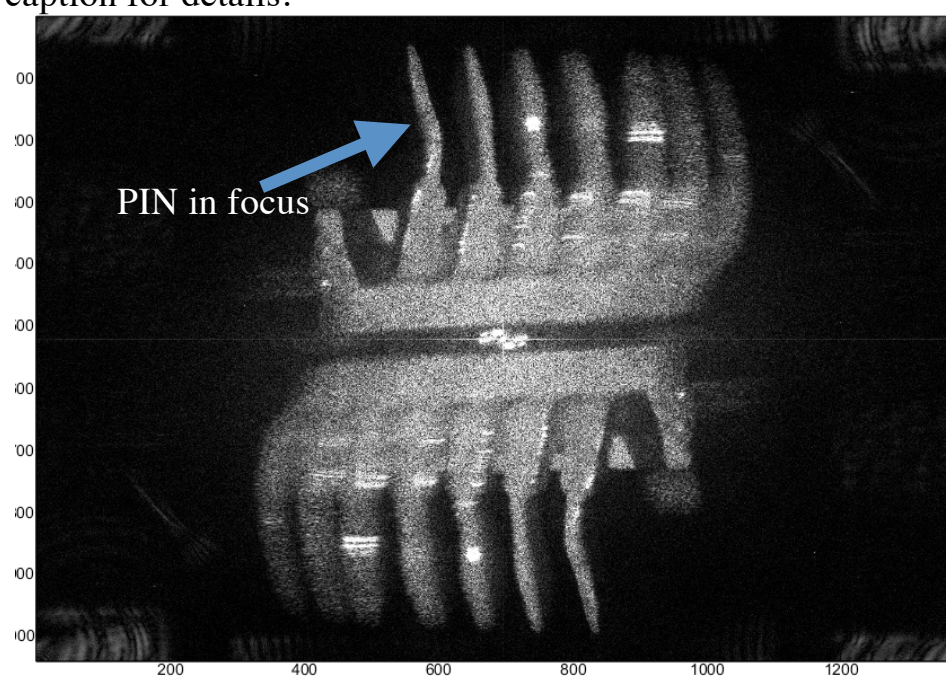

Fig. 3. Numerical reconstruction of a digital hologram captured using a Fourier geometry. Both the twin and real image terms are in focus. The DC term is roughly mapped to a spot in the center of the digital reconstruction. Note that the first PIN is in sharp focus.

To focus on a different section of the Fourier hologram we now change the value of $z_{\mathrm{R}}$ in Eq. (4) by multiplying with a numerical quadratic phase factor and perform a Fourier transform on the result (a similar approach is also discussed in Ref. [4]). Note the increased blurring of the DC and twin terms in Fig. 4 when compared to Fig. 3, also note the change in focus. 


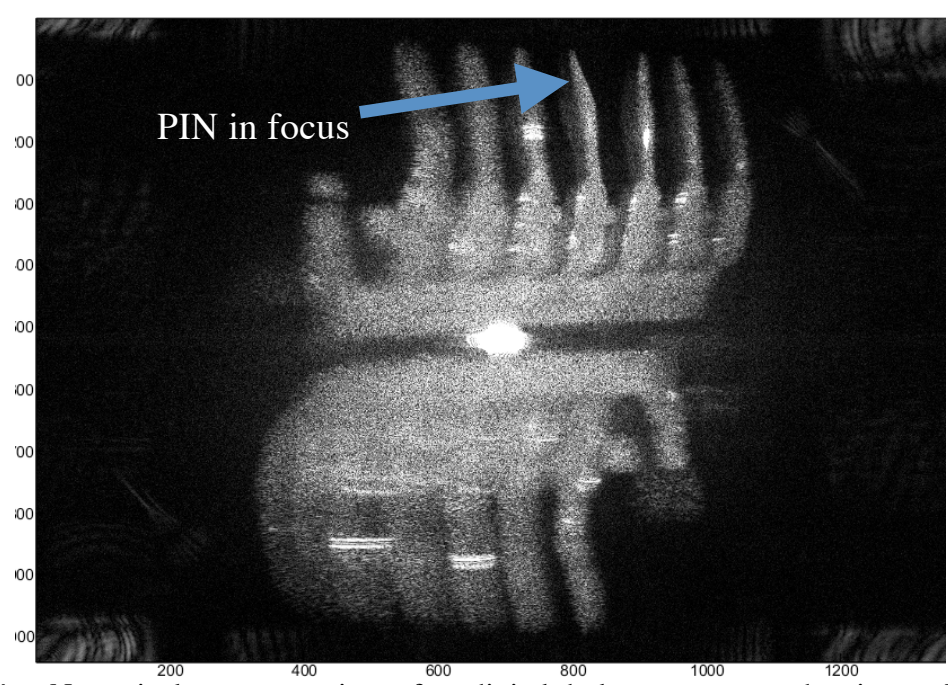

Fig. 4. Numerical reconstruction of a digital hologram captured using a Fourier geometry. Here we have re-focused using a numerical algorithm. The effect of the focusing is to blur the twin term and smear the DC term.

\section{Conclusion}

We have shown that both Fourier and Fresnel holograms can be refocused using numerical techniques however this focusing operation will effect the twin and DC terms differently in each case.

\section{References}

1. Kreis M. T, (2002) Frequency analysis of digital holography. Optical Engineering 41:771-778

2. Kelly P. D, Hennelly M. B, Pandey N, Naughton J. T, Rhodes T. W, (2009) Resolution limits in practical digital holographic systems. Under Review Optical Engineering

3. Kelly P. D, Hennelly M. B, Rhodes T. W, Sheridan T. J, (2006) Analytical and numerical analysis of linear optical systems. Optical Engineering 088201:1-12

4. Pedrini G, Froening P, Tiziani J. H, Mendoza Santoya F, (1999) Shape measurement of microscopic structures using digital holograms. Optics Communications 164:257-268 